Method A nested case-control study was conducted in rural areas of Shahroud, in the central region of Iran. We selected 65 child deaths from the 2000-2008 birth cohort of 10912 living newborns. A risk set sampling method was used to select controls (Case-Control ratio 1:2) who matched the cases in terms of age. The data were analysed using univariate and multivariate conditional logistic regression methods.

Results Significant associations with mortality were seen for: breastfeeding (OR $0.86,95 \%$ CI 0.79 to $0.93, p<0.001$ ), number of child cares (OR 0.90, 95\% CI 0.83 to $0.98, \mathrm{p}=0.017$ ) and low birth weight (OR 7.38, 95\% CI 1.38 to 39.58, $\mathrm{p}=0.020$ ).

Conclusion Incomplete breast feeding duration, insufficient number of child cares and low birth weight are important risk factors for 1-59 month child mortality in Iran. It appears that complex and multiple factors are involved in mortality of under-5-year-old children, so combined efforts will need to be applied to improve child health.

\section{P2-382 ILLEGAL STAY AND PRENATAL CARE IN IMMIGRANT PREGNANT WOMEN LIVING IN PORTUGAL}

doi:10.1136/jech.2011.142976l.12

E Coutinho, C Pereira, ${ }^{*}$ A Silva, J Duarte, N Veiga, P Nelas, M Ferreira, C Chaves. Cl \& DETS, Health School - Polytechnic Institute of Viseu, Viseu, Portugal

Background Immigration from low income countries represents a recent and increasing reality in Portugal. The legalisation process is slow and the illegal stay may represent a barrier to healthcare access. The objective of this study was to relate the illegal stay condition with the mother's obstetric surveillance.

Participants and Methods The sample included 499 immigrants living in Portugal, consecutively selected during the year 2010 in 21 Portuguese maternity hospitals. Data were collected in a face-to-face interview on the second day after delivery and with the consultation of medical hospital records. We considered adequate obstetric surveillance (AOS) if the mother had six or more appointments during pregnancy and the first occurred during the first trimester.

Results In this sample, the proportion of illegal immigrants was $10.7 \%$. The prevalence of AOS was $73.5 \%$, significantly lower in mothers in illegal situation $(59.1 \%$ vs $77.9 \%, p<0.01)$. AOS was associated with maternal age ( $\leq 25$ years $=57.3 \%, \quad 26-30$ years $=78.9 \%,>30$ years $=79.9 \%, p<0.01)$, women's education $(<6$ years $=60.6 \%, \quad 7-12$ years $=65.3 \% \quad>12$ years $=90.9 \%, \quad p<0.01)$, marital status (single $/$ divorced $=62.0 \%$, married $=79.9 \%, p<0.01$ ), profession (white collar $=84.5 \%$, blue collar $=68.3 \% p<0.01$ ) wanted pregnancy (yes $=81.4 \%, \mathrm{no}=50.5 \%, \mathrm{p}<0.01$ ). After adjustment by non conditional logistic regression for mothers age, education and marital status, the illegal stay is associated with $\mathrm{AOS}(\mathrm{OR}=0.48$; 95\% CI 0.29 to 0.82 )

Conclusion Among immigrant pregnant women living in Portugal, the AOS is associated with illegal stay condition.

\section{P2-383 LIFETIME FOLLOW-UP OF MYCOBACTERIUM TUBERCULOSIS INFECTED INDIVIDUALS INCLUDING THOSE WITH SUBSEQUENT HIV INFECTION}

\section{doi:10.1136/jech.2011.142976l.13}

\begin{abstract}
${ }^{1,2} \mathrm{~A}$ Crampin, ${ }^{*}{ }^{1} \mathrm{H}$ Longwe, ${ }^{1} \mathrm{D}$ Mulawa, ${ }^{1} \mathrm{~L}$ Mwaungulu, ${ }^{2} \mathrm{~J}$ Glynn, ${ }^{2} \mathrm{H}$ Dockrell, ${ }^{2} \mathrm{~N}$ French, ${ }^{2} \mathrm{P}$ Fine. ${ }^{1}$ Karonga Prevention Study, Chilumba, Malawi; ${ }^{2}$ London School of Hygiene and Tropical Medicine, London, UK
\end{abstract}

Introduction The assumption that infection with $M$ tuberculosis is life-long in the absence of treatment underlies the "statistic" that one third of people worldwide are currently infected. Exploration of the validity of this statement and the contribution of this reservoir to later disease are critical, given the WHO target of elimination of TB by 2050. HIV-positive individuals are assumed to be at high risk of "remotely" acquired $M$ tuberculosis infection when their immunity declines. Understanding individuals who clear or control infection is key to identifying correlates of protection and requires a well defined long term epidemiological cohort.

Methods A cohort of 2000 individuals with strong evidence of $M$ tuberculosis infection (tuberculin skin test [TST] $>20 \mathrm{~mm}$ without active disease at baseline) in Malawi were followed up after 25 years. Outcomes (active TB, death, departure) were recorded. A sample of survivors were investigated for HIV status and CD4 count, TST and commercial interferon gamma release assay (T-Spot. TB). A sub-sample were re-tested immunologically after isoniazid treatment.

Results Life-time risk of active TB was approximately $8 \%$, with highest incidence in the first 2 years. We identified groups who appear to have resolved infection: HIV-positive immunocompromised survivors with no evidence of $\mathrm{TB}$, and HIV-negative individuals with complete absence of characteristic immunological responses. Some individuals showed exaggerated immune response indicating ongoing immune stimulation.

Conclusions Latent $M$ tuberculosis infection may resolve without treatment and natural, identifiable, "protected" phenotypes may exist. These findings have implications for both control of the reservoir of latent infection and the potential for evaluation of vaccines.

\section{P2-384 RISK OF PRETERM BIRTH: A CASE-CONTROL POPULATION-BASED}

doi:10.1136/jech.2011.142976l.14

${ }^{1} \mathrm{P} L$ de Assunção, ${ }^{*}{ }^{1} \mathrm{H}$ M D Novaes, ${ }^{1} \mathrm{G}$ P Alencar, ${ }^{2} \mathrm{~A} S$ de Oliveira Melo, ${ }^{1} \mathrm{M} F$ de Almeida. ${ }^{1}$ University of São Paulo, São Paulo, São Paulo, Brazil; ${ }^{2}$ Institute of Integral Medicine Prof. Fernando Figueira, Recife, Pernambuco, Brazil

The prevalence of preterm birth has increased in recent years and is a worldwide public health problem. To study the risk factors for preterm birth we examined hospital deliveries in residents in the city of Campina Grande/PB, Brazil. The case-control populationbased study was conducted from June 2008 to May 2009. The medical data and interviews of mothers of 341 preterm births (cases) and 424 controls were conducted. Logistic multiple regression model, based on a hierarchised conceptual modelling, approach was performed. The risk factors for preterm birth were: previous preterm birth ( $\mathrm{OR}=2.32 ; 95 \% \mathrm{CI} 1.25$ to 4.29$)$, inadequate prenatal care (category II - three or more negative prerequisites) $(\mathrm{OR}=2.15$; $95 \%$ CI 1.40 to 3.27$)$, inadequate maternal weight gain $(\mathrm{OR}=2.33$; $95 \%$ CI 1.45 to 3.75 ), physical damage to the mother during pregnancy $(O R=2.10 ; 95 \%$ CI 1.22 to 3.60$)$, hypertension pressure $(\mathrm{OR}=17.08 ; 95 \%$ CI 3.67 to 79.43 ), hospitalisation during pregnancy $(\mathrm{OR}=5.64 ; 95 \% \mathrm{CI} 3.47$ to 9.15$)$, amniotic fluid volume changes $(\mathrm{OR}=2.28 ; 95 \% \mathrm{CI} 1.32$ to 3.95$)$, vaginal bleeding $(\mathrm{OR}=1.54 ; 95 \%$ CI 1.01 to 2.34$)$ and multiple gestation $(\mathrm{OR}=22.65$; $95 \%$ CI 6.22 to 82.46). The per capita income less than the minimum wage was a protective factor $(\mathrm{OR}=0.63$; $95 \%$ CI 0.39 to 0.99). The risk factors were similar to those observed in other national and international studies, except for the result of varying socioeconomic level. The high prevalence of poverty and low educational level, higher than in studies in the South in both outcomes, may have contributed to this result. Further studies are needed on the complexity of causal chains in preterm delivery in different contexts and differentiation by subtypes (spontaneous and indicated pre-term births). 